\title{
Geranylgeranylacetone protects against myocardial ischemia and reperfusion injury by inhibiting high-mobility group box 1 protein in rats
}

\author{
NENG WANG, XINWEN MIN, DONGFENG LI, PEIGEN HE and LIBIN ZHAO \\ Department of Cardiology, Dongfeng Hospital of Hubei Medical University, Shiyan, Hubei 442008, P.R. China
}

Received September 9, 2011; Accepted October 31, 2011

DOI: $10.3892 / \mathrm{mmr} .2011 .666$

\begin{abstract}
The high mobility group box 1 (HMGB1) protein plays an important role in myocardial ischemia and reperfusion (I/R) injury. Geranylgeranylacetone (GGA), a heat shock protein 72 inducer, has been reported to reduce myocardial I/R injury. The aim of this study was to investigate the cardioprotective mechanism of GGA during myocardial I/R injury in rats. Anesthetized male rats were treated once with GGA $(200 \mathrm{mg} /$ $\mathrm{kg}$, p.o.) $24 \mathrm{~h}$ before ischemia, and subjected to ischemia for $30 \mathrm{~min}$, followed by reperfusion for $4 \mathrm{~h}$. Lactate dehydrogenase (LDH), creatine kinase (CK), malondialdehyde (MDA), superoxide dismutase (SOD) activity and infarct size were measured. HMGB1 expression was assessed by immunoblotting. The results showed that pre-treatment with GGA $(200 \mathrm{mg} / \mathrm{kg})$ significantly reduced the infarct size and the levels of LDH and $\mathrm{CK}$ after $4 \mathrm{~h}$ of reperfusion (all $\mathrm{P}<0.05$ ). GGA also significantly inhibited the increase in MDA levels and the decrease in SOD levels (both $\mathrm{P}<0.05$ ). Meanwhile, GGA considerably suppressed the expression of HMGB1 induced by I/R. The present study suggests that GGA is capable of attenuating myocardial $I / R$ injury by inhibiting HMGB1 expression.
\end{abstract}

\section{Introduction}

The high mobility group box 1 (HMGB1) protein is a nuclear protein released by necrotic, apoptotic or activated innate immune cells (such as macrophages and monocytes) $(1,2)$, and serves as a novel pro-inflammatory cytokine in cardiovascular diseases (3-5). HMGB1 has recently been found to function as an early mediator of inflammation and cell injury during myocardial ischemia and reperfusion $(\mathrm{I} / \mathrm{R})$ as well as a classical early pro-inflammatory cytokine, such as tumor necrosis factor- $\alpha$ (TNF- $\alpha$ ) and interleukin-6 (IL-6), and may promote the release of TNF- $\alpha$ and IL-6. By contrast, HMGB1 A box

Correspondence to: Dr Neng Wang, Department of Cardiology, Dongfeng Hospital of Hubei Medical University, Shiyan, Hubei 442008, P.R. China

E-mail: wangneng0701@163.com

Key words: geranylgeranylacetone, high-mobility group box 1 protein, myocardial ischemia and reperfusion peptide (a specific HMGB1 antagonist) reduces myocardial I/R injury and inhibits the release of TNF- $\alpha$ and IL-6 $(6,7)$. These results suggest that HMGB1 plays a key role in myocardial I/R injury.

Geranylgeranylacetone (GGA), a heat shock protein 72 (HSP72) inducer, has been reported to reduce myocardial I/R injury $(8,9)$. However, the cardioprotective mechanism of GGA during myocardial I/R remains unclear. We hypothesized that GGA may reduce myocardial I/R injury by inhibiting HMGB1 expression. The main aim of this study was to investigate whether GGA protects against myocardial I/R injury by inhibiting HMGB1 expression in a rat myocardial I/R model.

\section{Materials and methods}

Animal preparation. The experiment protocol conformed to the Guideline for the Care and Use of Laboratory Animals published by the US National Institutes of Health (NIH Publication, revised 1996) and was approved by the Institutional Animal Care and Use Committee. Fifty male Sprague-Dawley rats (250-300 g) were randomly assigned into four groups receiving the following treatments: Group 1, sham operated control (SO) $(n=10)$; rats were subjected to surgical manipulation without the induction of myocardial ischemia. Group 2, SO + GGA (SO-GGA) $(n=10)$; rats were treated with GGA (200 mg/kg, p.o.; Sigma-Aldrich Co., St. Louis, MO, USA) (8) and then subjected to surgical manipulation without the induction of myocardial ischemia. GGA was dissolved in an emulsion with $5 \%$ gum arabic and $0.008 \%$ tocopherol. Group 3, ischemia and reperfusion $(\mathrm{I} / \mathrm{R})(\mathrm{n}=15)$; rats were subjected to the left anterior descending coronary artery (LAD) occlusion for $30 \mathrm{~min}$ followed by reperfusion for $4 \mathrm{~h}$. Group 4, GGA + I/R (GGA-I/R) (n=15); rats were treated with GGA $(200 \mathrm{mg} / \mathrm{kg}$, p.o.) $24 \mathrm{~h}$ prior to ischemia.

After being anesthetized with sodium pentobarbital (45 $\mathrm{mg} / \mathrm{kg}$, i.p.), the rats were ventilated artificially with a volume-controlled rodent respirator at 70 strokes per min. Rats were placed on an electric heating pad to maintain body temperature at $37^{\circ} \mathrm{C}$. Heparin (200 IU/kg, i.v.) was administered prior to ischemia. Lead-II of the electrocardiogram was monitored with subcutaneous stainless steel electrodes. The electrocardiogram was monitored using a computer-based EP system (LEAD2000B; Jinjiang Ltd., Chengdu, China). 
A thoracotomy through a left parasternal incision was performed to expose the anterior wall of the left ventricle. A 4-0 silk suture on a small curved needle was passed through the myocardium beneath the middle segment of the LAD branch, coursing down the middle of the anterior wall of the left ventricle. A small vinyl flake was passed into both ends of the suture, which was then fixed by clamping the tube with a mosquito haemostat. A successful myocardial I/R model was confirmed by ST segment elevation in Leads-II and regional cyanosis of the myocardial surface. The rats underwent a 30-min occlusion of the LAD, followed by a 4-h reperfusion.

Assessment of myocardial injury. To assess the lactate dehydrogenase (LDH) and creatine kinase (CK) levels, blood samples were collected and centrifuged. Standard techniques using commercialized assay kits were used for analyses, according to the manufacturer's instructions (Nanjing Jiancheng Bioengineering Institute, Nanjing, China). Values were expressed in international units (IU) per liter.

Assessment of infarct size. Infarct size was established by 2,3,5-triphenyltetrazolium chloride staining (TTC; SigmaAldrich), as previously described (7). Briefly, after reperfusion the LAD was occluded again and $2 \mathrm{ml}$ of $1.0 \%$ Evans blue dye was injected via the femoral vein. Each heart was then sliced horizontally to yield five slices. The slices were incubated in $1 \%$ TTC for $15 \mathrm{~min}$ at $37^{\circ} \mathrm{C}$. The infarct area (white) and the area at risk (red and white) from each section were measured using an image analyser (Image-Pro Plus 3.0; Media Cybernetics, Silver Spring, MD, USA). Infarct size was expressed as a percentage of the risk area volume (\%, infarct size/risk area).

Measurement of myocardium malondialdehyde (MDA) and superoxide dismutase (SOD) activity assay. MDA concentration and SOD activity in myocardial tissue were measured using commercialized assay kits, according to the manufacturer's instructions (Nanjing Jiancheng Bioengineering Institute), as described in a previous study (7). MDA concentration was used as an index of oxygen-free radical and SOD activity as the lipid superoxide level in the myocardium.

Immunoblotting analysis. Pulverized frozen ischemia areas of left ventricle samples were analyzed by quantitative immunoblotting using the HMGB1 antibody (Santa Cruz Biotechnology, Santa Cruz, CA, USA), as previously described (7). The expression of the protein was normalized to glyceraldehyde3-phosphate dehydrogenase (GAPDH) expression.

Statistical analysis. Statistical analysis was performed with the SPSS 14.0 (SPSS Inc., Chicago, IL, USA). All values were expressed as the means $\pm \mathrm{SD}$. The Student's t-test was used for between-group comparisons. One-way ANOVA or Welch was used for comparisons among groups, and the StudentNeuman-Keuls or Dunnett T3 was used for post-hoc multiple comparisons. Statistical significance was defined as $\mathrm{p}<0.05$.

\section{Results}

Infarct size. After $4 \mathrm{~h}$ of reperfusion, the infarct size induced by myocardial I/R was decreased by GGA pre-treatment

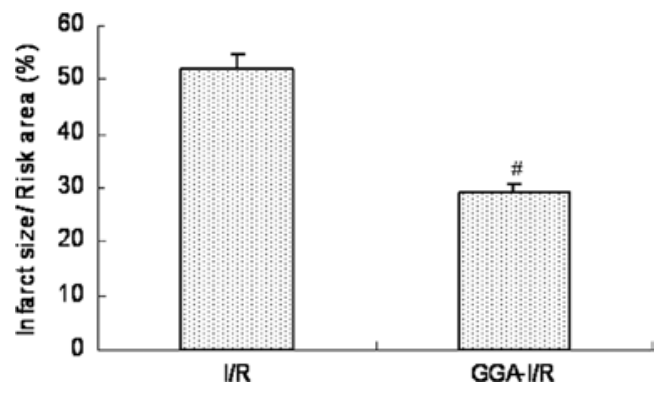

Figure 1. Effect of GGA on infarct size during I/R ( $=5) .{ }^{\#} \mathrm{p}<0.05$ vs. the $\mathrm{I} / \mathrm{R}$ group. GGA, geranylgeranylacetone; I/R, ischemia and reperfusion.

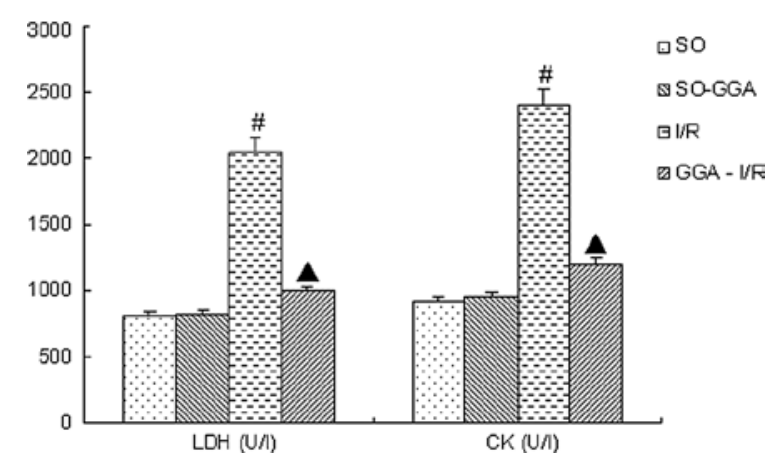

Figure 2. Effect of GGA on LDH and CK levels during I/R ( $\mathrm{n}=10) .{ }^{*} \mathrm{p}<0.05$ vs. the SO group; ${ }^{\wedge} \mathrm{p}<0.05$ vs. the I/R group. $\mathrm{LDH}$, lactate dehydrogenase; $\mathrm{CK}$, creatine kinase; SO, sham operated control; GGA, geranylgeranylacetone; $\mathrm{I} / \mathrm{R}$, ischemia and reperfusion.

compared to that in the I/R group ( $29.4 \pm 4.1$ vs. $52.2 \pm 4.9 \%$; $\mathrm{p}<0.05)$ (Fig. 1).

LDH and CK levels. After $4 \mathrm{~h}$ of reperfusion, both LDH and $\mathrm{CK}$ levels in the $\mathrm{I} / \mathrm{R}$ group were significantly increased compared to those in the SO and SO-GGA groups $(\mathrm{p}<0.05)$. However, the increasing levels of LDH and CK were significantly attenuated by GGA (both $\mathrm{p}<0.05$ ) (Fig. 2).

$M D A$ and SOD levels. After $4 \mathrm{~h}$ of reperfusion, MDA levels in the I/R group were significantly increased, while SOD levels were significantly decreased compared to those in the $\mathrm{SO}$ and SO-GGA groups $(\mathrm{p}<0.05)$. Both the increase in MDA levels and the decrease in SOD levels were significantly inhibited by GGA (both p<0.05) (Fig. 3).

Effect of GGA on HMGB1 expression during I/R. As shown in Fig. 4, HMGB1 expression was markedly increased after $4 \mathrm{~h}$ of reperfusion $(\mathrm{p}<0.05)$, which was significantly inhibited by GGA $(\mathrm{p}<0.05)$.

\section{Discussion}

The present study demonstrated that GGA is capable of reducing myocardial I/R injury and inhibiting HMGB1 expression. These results indicate that the cardioprotective effect induced by GGA during myocardial I/R injury may be associated with the inhibition of HMGB1 expression. 

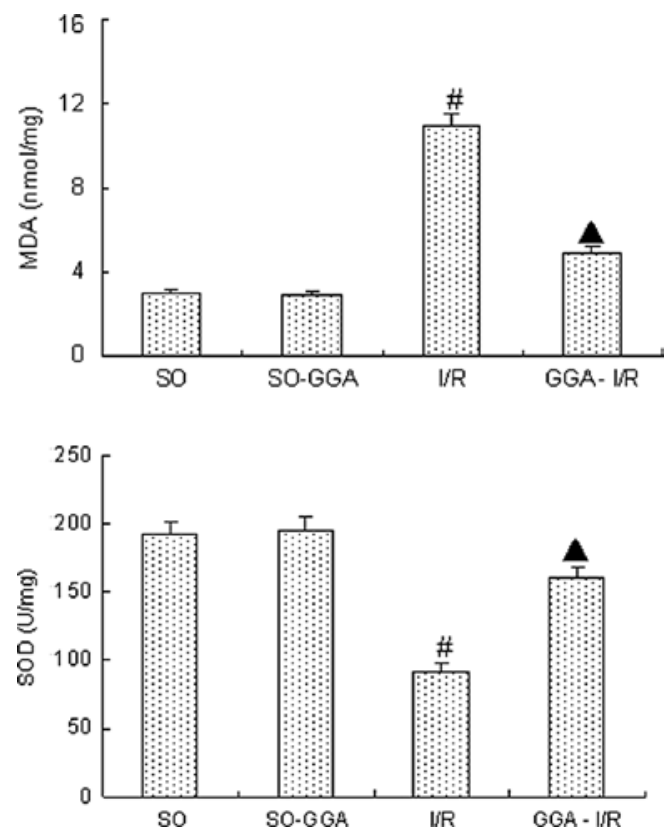

Figure 3. Effect of GGA on MDA and SOD during $\mathrm{I} / \mathrm{R}(\mathrm{n}=5) .{ }^{*} \mathrm{p}<0.05$ vs. the SO group; ${ }^{\wedge} \mathrm{p}<0.05$ vs. the I/R group. MDA, malondialdehyde; SOD, superoxide dismutase; SO, sham-operated control; GGA, geranylgeranylacetone; $\mathrm{I} / \mathrm{R}$, ischemia and reperfusion.
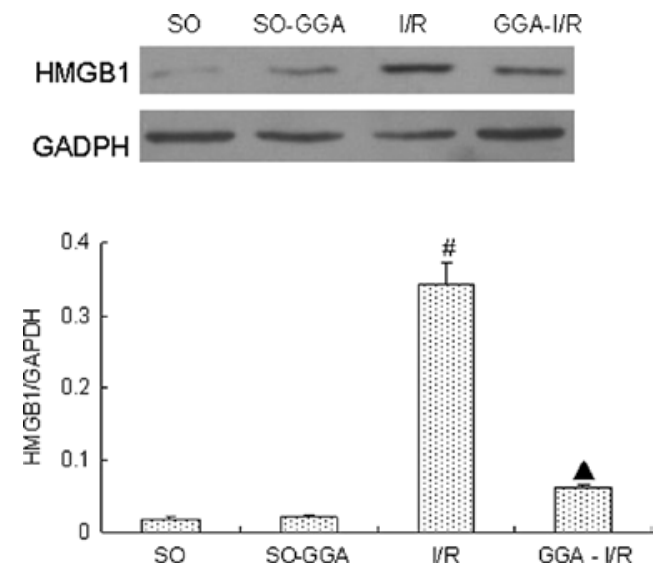

Figure 4. Effect of GGA on HMGB1 expression during I/R ( $=5)$. ${ }^{\#} \mathrm{p}<0.05$ vs. the SO group; ${ }^{\wedge} \mathrm{p}<0.05$ vs. the I/R group. HMGB1, high mobility group box 1 protein; GAPDH, glyceraldehyde-3-phosphate dehydrogenase; SO, sham operated control; GGA, geranylgeranylacetone; I/R, ischemia and reperfusion.

The cardioprotective effect induced by GGA during myocardial I/R injury has been demonstrated in previous studies $(8,9)$, but the mechanism remains unclear. Although our findings were consistent with those of previous studies, our results further indicated that GGA inhibits HMGB1 expression during myocardial I/R. Hu et al (7) recently demonstrated that HMGB1 promotes the apoptosis of myocytes and plays a significant role in myocardial I/R injury. Thus, inhibition of I/R-induced HMGB1 expression by GGA may suppress myocardial tissue apoptosis and reduce myocardial I/R injury. Moreover, previous studies showed that GGA promotes the expression of HSP72 $(8,9)$, while the activation of HSP72 inhibits the expression of HMGB1 $(10,11)$. We, therefore, speculated that GGA inhibits the expression of HMGB1 by promoting the expression of HSP72.

In the present study, GGA decreased the levels of MDA (one of the reactive oxygen species; ROS) and increased the levels of SOD (a key antioxidant enzyme). Previous studies indicate that ROS may be involved in the release of proinflammatory cytokine HMGB1 (12). Tang et al (13) indicated that hydrogen peroxide, one of the ROS, stimulates macrophages and monocytes to actively release HMGB1. Tsung et al (14) further demonstrated that the release of HMGB1 from cultured hepatocytes was also found to be an active process regulated by ROS. Recently, Loukili et al (15) showed that peroxynitrite, a potent cytotoxic oxidant, was involved in the release of HMGB1 in cardiac cells. Hence, inhibiting ROS may cause suppression of HMGB1 expression. In addition, Jiang et al (16) showed that HSP pre-treatment inhibits the apoptosis of the myocardium induced by hydrogen peroxide, which may promote the release of HMGB1. Consequently, these results suggest that GGA, an HSP72 inducer, protects against myocardial I/R injury by inhibiting HMGB1 expression.

Study limitations. In this study, we only observed the effect of GGA on HMGB1 expression during myocardial I/R. However, the precise mechanisms underlying our observations require further elucidation.

In conclusion, the present study suggests that GGA is capable of reducing myocardial I/R injury, which may, possibly, be associated with the inhibition of HMGB1 expression during myocardial I/R.

\section{References}

1. Scaffidi P, Misteli T and Bianchi ME: Release of chromatin protein HMGB1 by necrotic cells triggers inflammation. Nature 418: 191-195, 2002.

2. Bell CW, Jiang W, Reich CF and Pisetsky DS: The extracellular release of HMGB1 during apoptotic cell death. Am J Physiol Cell Physiol 291: C1318-C1325, 2006.

3. Hu X, Jiang H, Bai Q, et al: Increased serum HMGB1 is related to the severity of coronary artery stenosis. Clin Chim Acta 406: 139-142, 2009.

4. Wang LJ, Lu L, Zhang FR, Chen QJ, de Caterina R and Shen WF: Increased serum high-mobility group box-1 and cleaved receptor for advanced glycation endproducts levels and decreased endogenous secretory receptor for advanced glycation endproducts levels in diabetic and non-diabetic patients with heart failure. Eur J Heart Failure 13: 440-449, 2011.

5. Hu X, Zhou W, Bai Q, et al: Increased serum high mobility group box 1 protein in patients with atrial fibrillation. Biomed Aging Pathol 1: 52-55, 2011.

6. Andrassy M, Volz HC, Igwe JC, et al: High-mobility group box-1 in ischemia-reperfusion injury of the heart. Circulation 117: 3216-3226, 2008.

7. Hu X,Zhou X, He B, et al: Minocycline protects against myocardial ischemia and reperfusion injury by inhibiting high mobility group box 1 protein in rats. Eur J Pharmacol 638: 84-89, 2010.

8. Ooie T, Takahashi N, Saikawa T, et al: Single oral dose of geranylgeranylacetone induces heat-shock protein 72 and renders protection against ischemia/reperfusion injury in rat heart. Circulation 104: 1837-1843, 2001.

9. Yamanaka K, Takahashi N, Ooie T, Kaneda K, Yoshimatsu H and Saikawa T: Role of protein kinase $\mathrm{C}$ in geranylgeranylacetoneinduced expression of heat-shock protein 72 and cardioprotection in the rat heart. J Mol Cell Cardiol 35: 785-794, 2003.

10. Tang D, Kang R, Xiao W, et al: Nuclear heat shock protein 72 as a negative regulator of oxidative stress (hydrogen peroxide)induced HMGB1 cytoplasmic translocation and release. J Immunol 178: 7376-7384, 2007. 


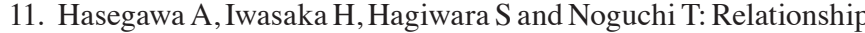
between HMGB1 and tissue protective effects of HSP72 in a LPS-induced systemic inflammation model. J Surg Res 169: 85-91, 2011.

12. Tang D, Kang R, Zeh HJ and Lotze MT: High-mobility group box 1, oxidative stress, and disease. Antioxid Redox Signal 14: 1315-1335, 2011.

13. Tang D, Shi Y, Kang R, et al: Hydrogen peroxide stimulates macrophages and monocytes to actively release HMGB1. J Leukocyte Biol 81: 741-747, 2007.

14. Tsung A, Klune JR, Zhang X, et al: HMGB1 release induced by liver ischemia involves Toll-like receptor 4-dependent reactive oxygen species production and calcium-mediated signaling. J Exp Med 204: 2913-2923, 2007.
15. Loukili N, Rosenblatt-Velin N, Li J, et al: Peroxynitrite induces HMGB1 release by cardiac cells in vitro and HMGB1 upregulation in the infarcted myocardium in vivo. Cardiovasc Res 89: 586-594, 2011

16. Jiang B, Xiao W, Shi Y, Liu M and Xiao X: Heat shock pretreatment inhibited the release of Smac/DIABLO from mitochondria and apoptosis induced by hydrogen peroxide in cardiomyocytes and C2C12 myogenic cells. Cell Stress Chaperones 10: 252-262, 2005. 